Research Article

Open Access

Tony Cheng

\title{
The Situational Structure of Primate Beliefs
}

\author{
DOI 10.1515/pipjp-2016-0006
}

\begin{abstract}
This paper develops the situational model of primate beliefs from the Prior-Lurz line of thought. There is a strong skepticism concerning primate beliefs in the analytic tradition which holds that beliefs have to be propositional and non-human animals do not have them (e.g., Davidson 1975, 1982). The response offered in this paper is twofold. First, two arguments against the propositional model as applied to other animals are put forward: an a priori argument from referential opacity and an empirical argument from varieties of working memory. Second, the Prior-Lurz situational model based on state of affairs as opposed to propositions is introduced and defended with two significant modifications. With this model of primate beliefs we can make progress in understanding how other primates can have certain mindreading capacity.
\end{abstract}

Keywords: beliefs; primates; mindreading; situational structure; state of affairs

\section{Animal Mindreading and Animal Minds}

Homo sapiens are minded beings. They are very likely mind-readers too, in the sense that they explain and predict (with confabulations) others' behaviors in terms of their grasps of others' mental states or episodes - doxastic states, motivational states, and so on. But what about other animals, in particular our primate relatives? More than three decades ago, Premack and Woodruff succinctly summarized relevant issues by asking, "Does the chimpanzee have a theory of mind" (1978)? In order to leave open various theoretical possibilities, we should not read too much into the word "theory" in this context, since it may turn out that some animals do read minds, but their relevant abilities do not consist in anything like a theory in some strict sense.

The answer to the question about primate mindreading conceptually presupposes the answer to the question concerning primate minds: what do primates' mental lives look like? Initially, one might naturally extend categories such as perception, belief, desire, emotion, and other familiar human mental categories to primates in understanding their minds. However, one salient difference between humans and other primates have to be taken into account - humans have language in the strict sense, while other primates do not. It is difficult to give a theoretical characterization of the distinctiveness of human languages that satisfies most people. Some philosophers appeal to compositional semantics, for example, but such an appeal is not without its opponents. ${ }^{1}$ For our purposes a principled answer to this question is not needed; suffice it to say that no other animals can talk or write about abstract affairs, things not directly related to animal strives, and so on. Human languages significantly shape human minds, so when we attempt to extend our model to other animals this huge difference has to be put in the center of focus. ${ }^{2}$

1 See, for example, Stephen Schiffer (2003).

2 In this paper my concern is only belief state and I do not assume that the account can be generalized to other mental states. However, if the structure of primate beliefs is indeed very different from human beliefs it is reasonable to assume that other mental states of primates are also less abstract and intellectual, as in the case of beliefs. Similarly, although I talk about "other primates" in this paper most studies I will refer to are about chimpanzees; I do not assume that what I argue here can be extended to, say, orangutans and monkeys.

*Corresponding author: Tony Cheng, University College London 
Examples of human full-blown beliefs include "I believe that it is going to rain very soon." Some languages do not have a grammatical equivalence to "that-clause" in English, but that is a superficial difference that can be ignored for present purposes. Human beliefs typically involve an assertoric, sentential structure, no matter what the surface grammatical structures look like. Now it should be clear that even if other primates do have something like beliefs, those states would presumably be very different from the human variety, since unlike humans, they do not have a language in the strict sense. However, mainstream analytic philosophy seems to be oblivious to this. Here I side with Dale Jamieson that "it is downright harmful if, after assimilating thinking to having a thought, we go on to suppose that having a thought is the same as having a propositional attitude" (2009: 15, italics mine). Donald Davidson's ingenious arguments against animal beliefs and belief attributions come to mind $(1975,1982)$. The next section deals with, and ultimately rejects, this idea that beliefs have to be propositional. A positive model will then be suggested.

\section{Against the Propositional and for the Situational}

The general form of the Davidsonian argument starts with certain distinctive features of human beliefs and argue that since other animals lack the crucial precondition of that kind of belief they have no belief whatsoever. Here a leap from human beliefs to beliefs in general is made. To be sure, it is possible that at the end of the day Davidson is right in thinking that the only format of belief is propositional, linguistic belief; but it is methodologically vicious if philosophers stick to a priori arguments so much that they do not bother paying attention to empirical findings and relevant conceptual clarifications at all (e.g., the possibility of other formats of belief). ${ }^{3}$ John McDowell (1996) extends this human-centred picture to perception and experience in general, which is perhaps even more controversial since experiences are supposed to be more primitive than beliefs. In his later writings, however, he seems to become sensitive to the fact that non-human animals have mental lives with very different formats. Evidence of this is his use of the term 'world-disclosing experience' to single out human experiences, leaving room for experiences in non-human animals with the Gadamarian distinction between world and environment, a detail that has no direct bearing to the present investigation. I shall not go into the details of these arguments here. In what follows I offer positive reasons for thinking that animals beliefs are non-propositional.

Again, human beliefs have the form "I believe that _.." One interesting feature of human belief attribution is that it is subject to what we might call the referential opacity constraint. Suppose that Lois believes that Superman can fly. Unbeknownst to her, Superman is in fact Clark Kent. Most people agree that it is problematic to attribute Lois the belief that "Clark Kent can fly." We can perhaps agree that in some sense she does have that belief, but to do this we need to employ a very special reading of the sentence, i.e., the so-called transparent use, according to which "Clark Kent" functions merely as a reference-fixer. Exactly how to understand the situation and how to solve the puzzle are huge topics in philosophy of language, but we do not need them here. What we need is the idea that this opacity does happen in human beliefs. One reason for opacity is that humans can think of things with many different "modes of presentation" or "guises," but we of course cannot know every possible way of talking about specific objects. With another terminology, human beliefs are fine-grained, at least according to a natural view in philosophy of language. It is not clear if this also applies to other animals. The ways they represent things are anchored to salient factors, for example, whether there are more dominant conspecifics around. ${ }^{4}$ This fits well with the account that will be developed later in this section. On that account, the objects of animal beliefs are states of affairs; compared to propositions that are composed by Fregean modes of presentation, states of affairs are coarse-grained in the sense that they are composed by objects and properties and not by modes of (re) presenting them (Lurz 2011: 134).

There are also empirical reasons for doubting that other primates' beliefs are propositional. Here I focus on recent works by Matsuzawa et al. on chimpanzees' working memory $(2007,2009)$. Their basic finding is that although chimpanzees might have worse memories in some respects they nevertheless "have an

3 For a more detailed discussion of a similar line of thought, see Robert Lurz (2011: 131-8).

4 Here I use the term 'represent' in a deflationary way; nothing hinges on it at this point, but more on this below. 
extraordinary working memory capacity for numeral recollection - better even than that of human adults tested in the same apparatus following the same procedure" (2007: 1004). I would say their description of chimpanzees' relevant capacities is too moderate; in fact, they are much better than both adult and young humans, as demonstrated in the above mentioned study by Matsuzawa et al.. ${ }^{5}$ In this experiment the subjects are six chimpanzees. They are all trained to use Arabic numerals, though five of them had no experience of using numerals in any task. The initial task consists in touching the numerals with the 1-2-3-4-5-6-7-8-9 sequence. Once being touched, the numeral in question will disappear, but this task is not very challenging since the rest of the numerals will not disappear until being touched. Although not difficult, one interesting fact is that chimpanzees can do it very quickly, much quicker than average human beings. ${ }^{6}$ It seems that they have different ways of retaining information. But what is really crucial is the later challenging task in which all other eight numerals (2-9) are masked after the first one (1) is touched. The subjects need to retain all the required information for several seconds, and when subjects start to perform the task they need to touch the screen by hand, an action which is distracting. But surprisingly enough, the chimps perform the task very well.

To see the difference between chimpanzees and humans in this respect, consider the famous Sperling Paradigm (1960) with humans as subjects. ${ }^{7}$ At the beginning of the experiments, subjects look at a blank screen with a fixation point in the middle of the screen. An array of letters constituting a grid then comes in as stimuli for 15-500 milliseconds. After the stimuli disappear, there will be a delay with a blank screen; the period of delay depends on specific experimental settings for different purposes. Then crucially, a cue tone comes in signifying which row the subjects are supposed to report (high tone for the high row, etc.). Since there is a delay between the stimuli and the cue, one might expect that the cue will not have any significant effect, since it comes too late. Surprisingly, however, subjects are actually very good at reporting the given row accurately, even though the cue comes much later than the stimuli.

Although human subjects are good at performing the task, what they can reliably report are only four letters or so. Comparing to the chimpanzee cases, it should be clear that humans' iconic working memory is much poorer. Granted, there are many differences in the two paradigms, so direct comparison of the two cases is not available. Still, it should be safe to say that the ways humans and chimpanzees store information are very different, and this is a good reason for thinking that if chimpanzees have beliefs, the formats of their beliefs should be very different. Notice how badly humans perform in the Matsuzawa task. One might want to restrict the difference to the perceptual level only, but it is a rather incredible picture that humans and chimpanzees have very different perceptual states but nevertheless their beliefs may possibly share a similar format. To be sure, this is by no means a deductive argument. What philosophy can take from empirical studies in general, I believe, is only evidence gesturing in certain directions. Now, some might argue that what is provided above is not even gesturing in the direction I am heading to. To this I reply that by itself it might not. Again, when scientists set out to identify research questions and conduct experiments, what they have in mind is very different from philosophers. For example, they do not think in terms of propositionality. By themselves empirical studies cannot replace philosophical arguments. In the present case, one might say that what were introduced above are about working memory performances, period. I do agree that the experiments themselves are about working memory and not beliefs, but if we can agree that the latter crucially rest on the former, then those experiments can be seen as indirectly relevant to our purposes. Beliefs are something we need to acquire and sustain through time which crucially involves working as well as other varieties of memories. To have a full theory of the relations between working memory and belief, of course, is beyond the scope the current investigation.

On the other hand, there are many other cognitive tasks that are easy for humans but difficult for chimpanzees. Chimpanzees are, for example, not good at "generalized imitation" (Myowa-Yamakoshi et al. 2004), "cross-modal matching” (Hashiya and Kojima 2001), "number concepts" (Wood et al. 2008), and so on. The point about number concepts is especially relevant here. Given that chimpanzees are so good at the

5 The experiment is available at the following https://www.youtube.com/watch?v=aAIGVT3N7BO.

6 See the clip from 0:35 on.

7 Sperling experiment:http://www.nyu.edu/gsas/dept/philo/faculty/block/demos/Sperling320msec.mov. 
task designed by Matsuzawa et al (2007), one might expect that they and their relative primates might be able to manage number concepts to some extent. However, as shown by Woods et al., rhesus monkeys are able to compare 1 and 2, 2 and 3, 3 and 4, but profoundly unable to do 4 and $5 / 3$ and 8 . This suggests that their grasp of numbers is not very good. Perhaps their working memory for abstract things is incomparable with their working memory for perceptually salient items. This is the main contention of the "trade-off theory" proposed by Matsuzawa:

At a certain point in evolution, because of limitations on brain capacity, the human brain may have acquired new functions in parallel with losing others - such as acquiring language while losing visuo-spatial temporal storage ability. (Matsuzawa 2009: 97).

And not surprisingly, number concepts go with language. How to work out the details of this trade-off story is of course difficult and controversial, but the general line seems to be promising. If so, we have further reasons to believe that beliefs in other primates are non-propositional, since the components of propositions are abstract concepts.

One might object that the above two arguments, if successful, only show that if animals have beliefs, those beliefs are non-propositional. But this is a big "if." Maybe what animals have are only "belief-like" states, but they are not identical to beliefs. This objection seems to be a fair one, and in response I do not propose a direct argument to the effect that what animals have, according to the above arguments, are beliefs. Rather, I propose to deflate the objection by arguing that this worry, though genuine, is orthogonal to my purposes. What is more interesting and significant, I submit, is to understand the kind of mental states and episodes non-linguistic animals possess and how those mental states and episodes cause their behaviors. Whether those mental things deserve to be called "belief" seems to be a judgment call. If one insists that only propositional states are worthy of the name, then I can concede that what animals have as argued above are only belief-like. However, I do think that a rather liberal use of the term "belief," is more fruitful. The reason is that what is crucial for belief is the assertoric force and its capacity to represent the world as being a certain way. Being propositional is our way of doing it. It does not follow that it is the only way of doing it.

To say primate beliefs are non-propositional invites the question about its nature - in particular, the challenge for us to characterize those beliefs in positive terms. Robert Lurz takes up this challenge in chapter 4 of his recent book (2011). In what follows I first briefly summarize his conception and then try to further develop the model.

Lurz credits Arthur Prior (1971) as a precursor of the conception. ${ }^{8}$ The Prior-Lurz model begins with the observation that belief relations do not have to be analyzed as

(a) $\mathrm{S} /$ believes/that $\mathrm{p}$,

but can be analyzed instead as

(b) S/believe that/p (2011: 133).

One initial worry is that the proposed analysis might get the structure of "that-clause" wrong. However, as I suggested at the beginning of the paper, we should not be too obsessed with the surface grammatical structure. What is important for the Prior-Lurz model is that the relatum on the right hand side is not an abstract proposition. For example, Lurz appeals to the notion of "states of affairs," possible states of the world (ibid.: 133). Although I follow the general line of the conception, I do not endorse the claim that states of affairs are "abstract entities" (ibid.). For one thing, the main virtue of states of affairs is that they are more concrete, hence it makes more sense to think that other animals can bear belief relations to them, at least on the face of it. As Lurz says, states of affairs consist of "individuals, properties, and relations." Given this, it is not clear to me that they are not concrete. To be sure, relations might themselves be abstract, but when animals perceive and think about certain relations between entities

8 It should be noted that in chapter 4 of his book Lurz explicitly states that this model is not an account about what animal beliefs are; rather, it is about what it is for animals to attribute beliefs to others. So this paper can be seen as an extension of the Prior-Lurz model. How plausible this extension depends on the following argumentations. 
and their properties, those relations are not perceived or thought as abstract. So it is more reasonable to hold that states of affairs are not abstract.

A worry about states of affairs is that their individuation conditions are not clear. For example, how many states of affairs are there in a given room? Are "that box is on the table" and "that table is under the box" different states of affairs? This is the general worry underlying the Fregean slingshot argument (1892). Or as Davidson puts it, if we really want to talk about "facts" (i.e., states of affairs that obtain), we can only talk about the "Great Fact" (Davidson 1969). However, the Prior-Lurz model does not commit to any specific ontology of facts or states of affairs. The notion of states of affairs can be defined relative to contexts, including the perceiving/thinking animal. For example, we can say that a certain chimpanzee at time $\mathrm{T}$ is in a belief relation with a state of affairs that consists of a dominant conspecific and its distance to certain foods. Of course, theoretically there is no principled way to single out the state of affairs in question, but that is not relevant, since only a few ways, or perhaps one way can be used to explain and predict that chimpanzee's subsequent behavior, and that is what justifies our belief attributions in question. Other idiosyncratic ways of individuating states of affairs are only in the eyes of theorists, not on the chimpanzee's mind. This partially hinges on Daniel Dennett's intentional stance idea (1988), and some might find this assumption problematic. For the purposes here, we do not endorse Dennett's theory, which has robust ontological import. Yet, I would recommend the Dennettian stance as at least a methodological guidance for belief attribution.

What is the main merit of the Prior-Lurz account? The crucial difference between propositions and states of affairs lies in their status and structures. States of affairs, unlike propositions, do not have sentential structures, and that is why we should use them to capture beliefs in non-linguistic animals, especially other primates for the present purposes. To give it a label, states of affairs have a situational or circumstantial structure. Non-linguistic animals do not think in abstract terms, since they lack the relevant apparatus. However, they are sensitive to environmental factors so that they can survive. An analogy would be visual thinking in mathematics (e.g., Giaquinto 2011). Some procedures in mathematics are so abstract that even sophisticated and well-trained humans find them difficult. However, if they visualize some items and their relations, it becomes easier to comprehend the procedure. To be sure, visuo-spatial thinking does not justify certain proofs in mathematics, but it is important for thinking. Similarly, as we have seen in the studies conducted by Matsuzawa et al., chimpanzees have strong visual working memory, so their belief states are likely to be more visual than abstract, and the relata of their beliefs are situational states of affairs, not abstract propositions.

Another possible difference between the view suggested in this article and Lurz's version is that while on his account primate beliefs are non-representational (Lurz 2011: 134) I am hesitant about this point. Lurz is right that "states of affairs, unlike propositions, are not by their nature representational entities" (ibid.). However, we have not ruled out the possibility that primates have something representational in mind and those items represent states of affairs. This representational account might have the virtue of making sense of the fact that non-linguistic animals also make mistakes in their beliefs. Indeed, even in some propositional accounts (for a prominent example see Tye 2000), propositions are thought as being represented, as opposed to things representing. That is, humans have a belief-relation to propositions. If so, then propositions and states of affairs are both things being represented. The crucial difference between them lies in their structures. It is fine to have different versions of non-propositional accounts, and here I do not argue for my variant that primate beliefs are representational. The point to emphasize is that the main disagreement between the propositional account and the account based on states of affairs concerns structures.

Another concern about the representational account is that it over-intellectualizes non-linguistic animals. A thrust of the Davidsonian argument is that non-linguistic animals cannot have concepts of belief and objective truth. The merit of the Prior-Lurz account is precisely to avoid this over-intellectualization. Now if we think that primate beliefs are representational (though non-propositional), don't we fall prey to Davidsonian objections again? I suggest that we go back to Quine's picture, in particular his use of "assent" and "dissent" (Quine 1960): these two notions are purely behavioral and involve only observable behaviors. This pair of notions is what will help us start making progress in radical translation. Davidson's criticism 
of this maneuver is that Quine's position cannot give us a systematic theory of language. Davidson's alternative is to use the idea of "holding true" to replace "assent," so that the concept of truth comes into play, and in turn brings in Tarski's recursive story (1967). However, this objection to Quine does not apply in the present context, since our subjects here are non-linguistic animals. Since they do not have linguistic abilities, the Davidson-Tarski theory does not apply to them. Quine's story has the merit that it does not over-intellectualize non-linguistic animals. One way to understand Davidson's objection to Quine is that Quine's picture is too primitive (i.e., "assent" as opposed to "holding true") to capture human discursive language; but if so by the same token Davidson's picture is too advanced or sophisticated to capture nonlinguistic animals' beliefs.

I shall end this section by considering a general worry of the Prior-Lurz picture. Since the situational structure is very different from the propositional one, one might be skeptical about whether we are doing belief-attribution at all when we operate with the situational structure. One initial response is that the attribution helps us explain and predict animals' behaviors, but this is not enough since other mental states, in particular perceptual experiences, can explain and predict behaviors too. My reply to this concern is that we can see the distinction between the occurrent and the dispositional in animals. It is true that in the study of Matsuzawa et al. quoted above, chimpanzees' behaviors are immediate after they see the arrays on screens, hence it is possible that beliefs do not operate there. However, in many other cases animals obviously have dispositional mental states and they behave according to those dispositional states. For example, in various experiments in which we have multiple chimpanzees and perhaps human experimenters, the chimpanzee subjects do not only act on their perceptual experiences, since at many points in those experiments there are occlusions between them and their conspecifics and other relevant items (e.g., Hare et al. 2000). Therefore, we have to admit that they have perceptual beliefs, as opposed to perceptual experiences only. ${ }^{9}$ One might object that the dispositions here might be purely behavioral, i.e., non-mental. This kind of countermove is almost always possible. I do not intend here to rule out the possibility that the relevant dispositions could be behavioral only. I have provided the case for the idea that at least some of those dispositions are mental, either perceptual or doxastic. More will be discussed on this in the next section.

\section{From One's Mind to Others' Minds}

Given this Prior-Lurz conception of primate beliefs, how should we proceed from here to a further question whether other primates, in particular chimpanzees, understand their conspecifics and perhaps human experimenters in terms of their understandings of others' mental states? Recall that we have confined ourselves to beliefs only; issues about other mental states, e.g., intention, perception, knowledge, have analogous but not exactly the same difficulties. In what follows I first outline the main problem briefly and provide some suggestions.

A natural starting point is the so-called "logical problem" (Lurz 2011: 25). It is logical rather than empirical, because it has an "in principle" flavor. In the case of humans, the situation is relatively simple because human subjects can give linguistic reports. Although the status of report is controversial, at least it is a prima facie way to know what human subjects have in mind. In the case of non-linguistic animals, however, the difficulty is more daunting, since they cannot report linguistically, and worse, we cannot give them linguistic descriptions about the experiments in question. As a result, we have to conduct experiments with behavioral cues. But if we do so, it seems impossible to tease apart behavior-reading and mind-reading

9 One important issue here is how different this picture is from the view of José Luis Bermúdez (2003). He starts with a "minimalist" account in which nothing is propositional-like, and raises various objections to it (34-63). Then he proposes a "success semantics" to strengthen the account (64-108). On the Prior-Lurz model, states of affairs are still structured in a significant sense, hence it is not clear that it will become the target of Bermúdez. However, here is not the place to go into his book-long project. What we have here should be compatible with the claim that the present account is not the whole story and various supplements are called for. What is more important for us to establish here is that propositional account does not work for the case of non-linguistic animals. 
hypotheses. It seems that we will always have a "complementary behavior-reading hypothesis" (ibid.: 34). For example, when mind-reading theorists use "seeing" to capture chimpanzees' understanding of others, it seems that their behavior-reading opponents can always use "direct line of gaze" to replace the notion. That is, it seems unavoidable that every mental term has its behavioral counterpart, and current experimental protocols seem to be unable to decide between the two hypotheses. ${ }^{10}$

What we need are new experimental protocols that are sensitive to the differences of the two empirical hypotheses. Lurz offers three of them towards the end of his book. As he says in conclusion:

Whether animals are capable of attributing beliefs...is very much an open empirical question...I have sketched three possible experimental protocols that can [potentially solve the problem]. The rest is now in the hands of empirical researchers and, of course, the animals. (Lurz 2011: 193, my italics)

Rather than repeating Lurz's proposal here, in what follows I shall consider possible responses to the Prior-Lurz account of situational structure of primate beliefs. This is at least an indirect contribution to the debate since in the past many contributed to the debate in terms of the propositional model only. Before actually testing their theories with Lurz's protocols, it is important to see what different theorists might say about the situational model of primate beliefs. For mind-reading theorists, they might want to say that since chimpanzees have good perceptual working memory but not good at abstract processing, even if they are mind-readers to some extent, they are not able to think of others in abstract terms. For example, in cases where we have a subordinate and a dominant chimpanzee, with foods behind occlusions so that the subordinate one sees that the dominant one does not have direct line of gaze to the foods in question, one assumption could be that the subordinate might hold a belief relation regarding the situation in a possibly pictorial way, where the situation includes the dominant chimpanzee's mental states. On the other hand, the behavior-reading theorists might suggest that given the good perceptual working memory, it makes sense to say that chimpanzees can detect complex behavioral cues from others and then store those pieces of information in mind. The crucial thing for the behavior-reading theorists is to insist that only behavioral terms are involved in the interactions. As said above, the controversy needs to be solved by running new experimental protocols; before that, one interim conclusion can be drawn which is that both mind-reading and behavior-reading hypotheses could at a preliminary level accommodate the Prior-Lurz model of primate beliefs. More details need to be added once new experiments based on new protocols are done.

I now turn to consider a more radical position in conclusion of this paper. The position that the dichotomy between mind-reading and behavior-reading hypotheses, though seemingly intuitive, does not really make empirical sense. ${ }^{11}$ The distinction is intuitively acceptable since one side is based on both mental terms and behavioral terms while the other is based on behavioral terms only. Povinelli's challenge is that although the two hypotheses are clearly distinct, no extant experimental paradigm can tease them apart. But the present skepticism attempts to go in the other direction: since no extant experimental paradigm can favor one hypothesis over the other, we should cast the very distinction into doubt. Perhaps the whole debate of animal mindreading is misguided by the distinction. The relevant discussions are substantial of course, but according to this position, we should conceptualize it in some other ways, if the distinction between the two hypotheses does not make good empirical sense.

It is interesting to try to turn the whole discussion around when we find ourselves stuck in quandaries for several decades. However, I do not find this particular proposal promising. First of all, there is a difference between relying on intuitions and on conceptual knowledge. Philosophers sometimes rely on intuitions in thinking about thought experiments and theories, but in the present case I submit that people accept the distinction between mind-reading and behavior-reading hypotheses not because of intuitions but because of semantic differences. One allows both behavior and mental terms but the other allows behavior terms only, period. This is not a use of intuition; this is our grasp of word meanings, including conjunction. To

10 The logical problem is often also referred as "Povinelli's challenge" (e.g., see his 1996). For detailed discussion of this problem, see Lurz (2011: ch.2), Cecilia Heyes (1998), and Call \& Tomasello (2008).

11 This position was defended by Kyle Ferguson in personal communication. In what follows I will characterize the position in my own terms. Needless to say, he is not responsible for anything I say here. 
account for our semantic grasping is difficult in philosophy of language but what we need here is not an account of it. What we need is only the relevant grasp of concepts invoked in those hypotheses. It is true that sometimes when theories become too complicated we lose our clear grasps of them and it might turn out that under careful reconstruction some theories are equivalent to each other. But this is not the case in this context, since both hypotheses are clear and simple enough. To insist that we have to operationalize first in order to maintain certain distinctions exemplifies an ideal but not realistic picture of science. Or even worse, it might embody a form of verificationism. Human capacities are quite limited, hence to think that nature's joints should be carved in terms of our operationalization abilities is to overestimate our position in nature. Operationalization should be a regulative ideal, not a starting point.

This completes my investigation in this paper. As a philosopher I do believe that cooperation between scientists and philosophers should be a standard enterprise and not a rare instance. Lurz has recently contributed to this interdisciplinary project to a great extent from the philosophical side; I hope in this paper I have made a rather humble but still visible contribution in the same vein.

\section{References}

Bermúdez, J. (2003). Thinking without words. Oxford: Oxford University Press.

Call, J. and Tomasello, M. (2008). Does the chimpanzee have a theory of mind? 30 years later," Trends in Cognitive Sciences, 12(5), 187-192.

Davidson, D. (1967). Truth and meaning. Reprinted in his Inquiries in truth and interpretation (2001). Oxford: Oxford University Press.

Davidson, D. (1969). True to the facts. Reprinted in his Inquiries in truth and interpretation (2001). Oxford: Oxford University Press.

Davidson, D. (1975). Thought and talk. Reprinted in his Inquiries in truth and interpretation (2001). Oxford: Oxford University Press.

Davidson, D. (1982). Rational animals. Reprinted in his Subjective, intersubjective, objective (2001). Oxford: Oxford University Press.

Dennett, D. (1988). The intentional stance. Cambridge, MA: MIT Press.

Fodor, J. (1975). The language of thought. Cambridge, MA: Harvard University Press.

Frege, G. (1892). On sense and reference. Reprinted in M. Beaney (ed.), The Frege reader (1997). Oxford: Wiley-Blackwell. Giaquinto, M. (2011). Visual thinking in mathematics: an epistemological study. Oxford: Oxford University Press.

Hare, B. et al. (2000). Chimpanzees know what conspecifics do and do not see. Animal Behavior, 59, 771-785.

Hashiya, K. and Kojima, S. (2001). Acquisition of auditory-visual intermodal matching-to-sample by a chimpanzee (Pan troglodytes): comparison with visual-visual intramodal matching. Animal Cognition, 4, 231-239.

Heyes, C. (1998). Theory of mind in nonhuman primates. Behavioral and Brain Sciences, 21, 101-148.

Inoue, S. and Matsuzawa, T. (2007). Working memory of numerals in chimpanzees. Current Biology, 17(23), 1004-1005.

Jamieson, D. (2009). What do animals think? In R. Lurz (ed.), The philosophy of animal minds. Cambridge, UK: Cambridge University Press.

Lurz, R. (2011). Mindreading animals. Cambridge, MA: MIT Press.

McDowell, J. (1996). Mind and world. Cambridge, MA: Harvard University Press.

McDowell, J. (2007). What myth? Reprinted in his The engaged intellect (2009). Cambridge, MA: Harvard University Press.

Matsuzawa, T. (2009). Symbolic representation of number in chimpanzees. Current Opinion in Neurobiology, 19, 92-98.

Myowa-Yamakoshi, M. and Matsuzawa T. (1999). Factors influencing imitation of manipulatory actions in chimpanzees. Journal of Comparative Psychology, 113, 128-136.

Myowa-Yamakoshi, M. et al. (2004). Imitation in neonatal chimpanzees (Pan troglodytes). Developmental Science, 7, $437-442$.

Povinelli, D. (1996). Chimpanzee theory of mind? The long road to strong inference. In P. Carruthers and P. Smith (eds.). Theories of theories of mind. Cambridge, UK: Cambridge University Press.

Premack, D. and Woodruff, G. (1978). Does the chimpanzee have a theory of mind? Behavioral and Brain Sciences, 1, 515-526. Prior, A. (1971). Objects of thought. Oxford: Oxford University Press.

Quine, W. V. (1960). Word and object. Cambridge, MA: MIT Press.

Schiffer, S. (2003). The things we mean. Oxford: Oxford University Press.

Sperling, G. (1960). The information available in brief visual presentations. Psychological Monographs: General and Applied, 74(11), 1-29.

Tye, M. (2000). Consciousness, color, and content. Cambridge, MA: MIT Press.

Wood, J. et al. (2008). Free-ranging rhesus monkeys spontaneously individuate and enumerate small numbers of non-solid portions. Cognition, 106, 207-221. 